\title{
Ecological and Human Health Risk Assessment of Toxic Metals in Water, Sediment and Fish from Lower Usuma Dam, Abuja, Nigeria
}

\author{
R. Wuana, C. Ogbodo*, A. U. Itodo, I. S. Eneji \\ Department of Chemistry and Centre for Agrochemical Technology \& Environmental Research (CATER), Federal University of \\ Agriculture, Makurdi, Nigeria \\ Email: *uniquechiogb@gmail.com
}

How to cite this paper: Wuana, R., Ogbodo, C., Itodo, A. U., \& Eneji, I. S. (2020). Ecological and Human Health Risk Assessment of Toxic Metals in Water, Sediment and Fish from Lower Usuma Dam, Abuja, Nigeria. Journal of Geoscience and Environment Protection, 8, 82-106. https://doi.org/10.4236/gep.2020.85006

Received: March 12, 2020

Accepted: May 12, 2020

Published: May 15, 2020

Copyright $\odot 2020$ by author(s) and Scientific Research Publishing Inc. This work is licensed under the Creative Commons Attribution International License (CC BY 4.0).

http://creativecommons.org/licenses/by/4.0/

\begin{abstract}
The study assessed the levels of some toxic metals (As, Cd, Cr, $\mathrm{Cu}, \mathrm{Hg}, \mathrm{Ni}, \mathrm{Pb}$ and $\mathrm{Zn}$ ) with their potential ecological and human health risks in water, African Catfish (Clarias gariepinus), Tilapia (Oreochromis spilurus niger) and sediment samples from the Lower Usuma dam FCT, Nigeria during two major seasons in a year (rainy and dry seasons). Toxic metal concentrations were determined using Atomic Absorption spectrophotometry $(\mathrm{Cd}, \mathrm{Cr}, \mathrm{Cu}$, $\mathrm{Ni}, \mathrm{Pb}$ and $\mathrm{Zn}$ ) and Atomic Emission Spectrophotometry (for As and $\mathrm{Hg}$ ), and the results obtained were compared with national and international standards. The ecological and human health risk indices of the toxic metals present in the samples from the Dam were evaluated and interpreted. Tilapia from the dam posed the highest but medium ecological and human health risk due to $\mathrm{Pb}$ concentration of up to $7.11 \mathrm{mg} / \mathrm{kg}$; ecological risk index of 35.55 and hazard quotient of 50.78. Overall ecological and human health risks were low due to the low concentrations of other toxic metals determined. As, $\mathrm{Cd}, \mathrm{Cr}$, $\mathrm{Cu}, \mathrm{Hg}, \mathrm{Ni}$ and $\mathrm{Zn}$ concentrations were all below WHO limits in the LUD water; $\mathrm{Ni}$ and $\mathrm{Pb}$ were above limits in the African Catfish and Tilapia samples. The data obtained were analyzed using one-way analysis of variance (ANOVA) and significant differences accepted at $p \leq 0.05$. There was no statistical difference in the concentrations of toxic metals in water but there was significant difference between the concentrations of toxic metals in the fish and sediment samples. Correlation was found to exist between toxic metals in the water, fish and sediment analyzed from the dam. The ecological and human health risks of toxic metals in Lower Usuma dam require regular checks and monitoring hence, it was recommended by the researcher, that this and similar research work be carried out annually by NESREA and also, as research work by other students of Environmental and Analytical chemistry.
\end{abstract}




\section{Keywords}

Toxic, Metals, AAS, AES, Concentration, Ecological Risk, Human Health Risk, Lower Usuma Dam, Contamination Factor, Water, Sediment, Fish

\section{Introduction}

A dam is a reservoir constructed for storage and impounding of water for domestic, agricultural and industrial uses by people living in a community or environment. Dams are also constructed to prevent floods, to supply drinking and domestic water, to generate energy and for irrigation purposes. The idea of dam construction or a water storage structure or a reservoir is intended for all year round water supply for domestic, irrigation and industrial uses and other recreational activities like fishing, swimming and navigational purposes (Umaru et al., 2014).

Three rivers transfer water into the Lower Usuma Dam, one of the known dams in Nigeria; the River Usuma, River Gidna and River Gurara (Gurara dam). Like an artificial lake that it is, the dam receives water all year round without any outflow, in addition to the concrete and metal components of the construction. The Gurara water transfer provides for the transfer of raw water from Gurara dam in Kaduna state to Lower Usuma dam in Federal Capital Territory (FCT), Abuja through a $75 \mathrm{~km}$ conduit pipeline to augment water supply to FCT as a result of rapid population growth. Besides this arrangement for dam-to-dam inflow, inlet of water from the other natural sources follows a natural course (Okunlola et al., 2014). Care must be taken, however, to ensure that such collection of water does not accumulate toxic substances from the diverse sources.

Heavy (Toxic) metal refers to any metallic chemical element that has a relatively high density and toxic or poisonous at low concentrations. They cannot be degraded or destroyed and can enter our bodies through food, drinking water and air. Heavy metals are dangerous because they tend to bioaccumulate. Bioaccumulation is an increase in the concentration of a chemical in a biological organism over time, compared to the chemical's concentration in the environment (Amadi, 2011). The role of trace metals in biochemical life processes of aquatic plants and animals and their presence in trace amounts in the aquatic environment are essential. However, at high concentrations, these trace metals become toxic (Jaishankar et al., 2014). Toxic metals in the aquatic environment exist in sediment, suspended particulate $(>0.45 \mu \mathrm{m})$, hydrous oxides and humic colloids, and in true solution (Morais et al., 2012).

Toxic metals are found naturally in the earth and may enter into the environment through mining activities, industrial discharge and from household applications. They become concentrated as a result of human activities and can enter plant, animal, and human tissues through inhalation, diet, and manual handling. Then, they can bind to and interfere with the functioning of vital cellular components. Dams are sinks for toxic metals that are continuously washed off from 
rocks and soils that are directly exposed to surface waters. The common sources of toxic metals are from dead and decomposing vegetation, animal matter, wet and dry fallouts of atmospheric particulate matters and from man's activities. The hazardous nature of heavy metals has been recognized because of their bioaccumulative nature in biotic systems.

This study seeks to determine the concentrations of toxic metals in the water, sediments and fishes living in the Lower Usuma dam Abuja, Nigeria, which local residents consume and assess the ecological and human health risk indices of such toxic metals.

\section{Materials and Methods}

\subsection{Materials}

\subsubsection{Reagents}

Only chemicals of analytical reagent grade (99.9\% purity) were utilized and they were obtained from reputable chemical distribution companies. The following reagents were used for the analyses: Trioxonitrate (V) acid (analytical grade), hydrogen peroxide, tetraoxosulphate (VI) salts of $\mathrm{As}, \mathrm{Cd}, \mathrm{Cr}, \mathrm{Pb}, \mathrm{Hg}, \mathrm{Zn}, \mathrm{Ni}$ and $\mathrm{Cu}$. Doubly distilled, de-ionized water was used in the preparation of all solutions in the experiments.

\subsubsection{Apparatus}

An AA 6800 SHIMADZU Atomic Absorption spectrophotometer (AAS) was used for $\mathrm{Cd}, \mathrm{Cr}, \mathrm{Cu}, \mathrm{Ni}, \mathrm{Pb}$ and $\mathrm{Zn}$ determinations while an Agilent 4210 MP-AES Atomic Emission Spectrophotometer (AES) was used for As and $\mathrm{Hg}$ determinations. Other pieces of apparatus used include, plastic sample bottles, measuring tape, electronic weighing scale, thermometer, Whatman No 41 filter paper, funnels, beakers, measuring cylinders, stirring rod, dropping pipette, standard volumetric flasks (for preparing reagents) and a time piece.

\subsubsection{Description of the Study Area}

The Lower Usuma dam is located within Ushafa community Abuja, between latitude $7^{\circ} 25^{\prime} 16^{\prime \prime} \mathrm{E}$ and longitude $9^{\circ} 01^{\prime} 12^{\prime \prime} \mathrm{N}$ and it covers a land mass of 2,500,000 $\mathrm{m}^{2}$. It is located at about the highest point of the Nigerian Federal Capital Territory, about $15 \mathrm{~km}$ northwest of Abuja on $570 \mathrm{~m}$ altitude. It is sited on a virgin location where human activity is minimal, thereby ensuring non pollution of the environment and freedom from industrial impurity. Though, pollution may be transported with the water from the contributory rivers.

The barrier system consists of two (2) dams: the main dam and the saddle dam. The main dam has a length of $1.3 \mathrm{~km}$ and a height of $47 \mathrm{~m}$, with a crown that is $10 \mathrm{~m}$ wide. The saddle dam is $470 \mathrm{~m}$ long and has a height of $15 \mathrm{~m}$. The lake has a width of $3.5 \mathrm{~km}$ and a length of $3 \mathrm{~km}$ and holds $1.2 \times 10^{8} \mathrm{~m}^{3}$ of raw water. The water from the Dam flows to the Lower Usuma dam waterworks, where the water is treated before being distributed to Abuja for diverse uses. The total capacity of the waterworks is $1.0 \times 10^{4} \mathrm{~m}^{3} / \mathrm{hr}$ (Ibrahim et al., 2014). 
The major occupation of the neighboring inhabitants is farming and hunting. The immediate area comprises of Clinton village, Ushafa kasa, Ushafa Sama and Ushafa new extension, while the entire community is made up of Cogo, Jigo, Pwambara, Peyi and Usuma dam quarters. Figure 1 shows a map of the study area and sampling points.

\subsection{Methods}

\subsubsection{Sampling and Digestion of Water}

Water sampling was done according to the procedure described by Ndimele and Kumolu-Johnson (2012). Water samples from the dam were randomly collected from twelve points at a depth of about $0.3 \mathrm{~m}$ below the water surface. Samples from four locations were homogenized and each put into three $500 \mathrm{ml}$ plastic bottles during the periods, July 2019 (Rainy season) and January 2020 (Dry season). Prior to sampling, the bottles were cleaned with $10 \%$ nitric acid and rinsed with distilled water. The bottles were then rinsed three times with the dam water at the time of sampling. Samples were collected by direct immersion of the sampling bottle into the dam. Immediately after sample collection, $2 \mathrm{~mL}$ nitric acid (AR grade) was added to the water samples to reduce adsorption of metals onto the walls of the plastic bottles. The sample bottles were then labeled to indicate date of sampling and the sampling site after which the samples were transported in an ice-box to the laboratory and stored at $4^{\circ} \mathrm{C}$ awaiting analysis. Digestion of the water samples was done in triplicate using concentrated nitric acid (Analytical Grade) according to the method described by Zhang (2007).

\subsubsection{Sampling and Digestion of Sediment Samples}

Sediment samples were taken from the bottom surface of the dam $(0-5 \mathrm{~cm}$ thick) using an Eckman grab according to Osman and Kloas (2010) during the periods, July 2019 (Rainy season) and January 2020 (Dry season). For each sample, three sediment grabs were randomly taken from the banks and mid-dam,

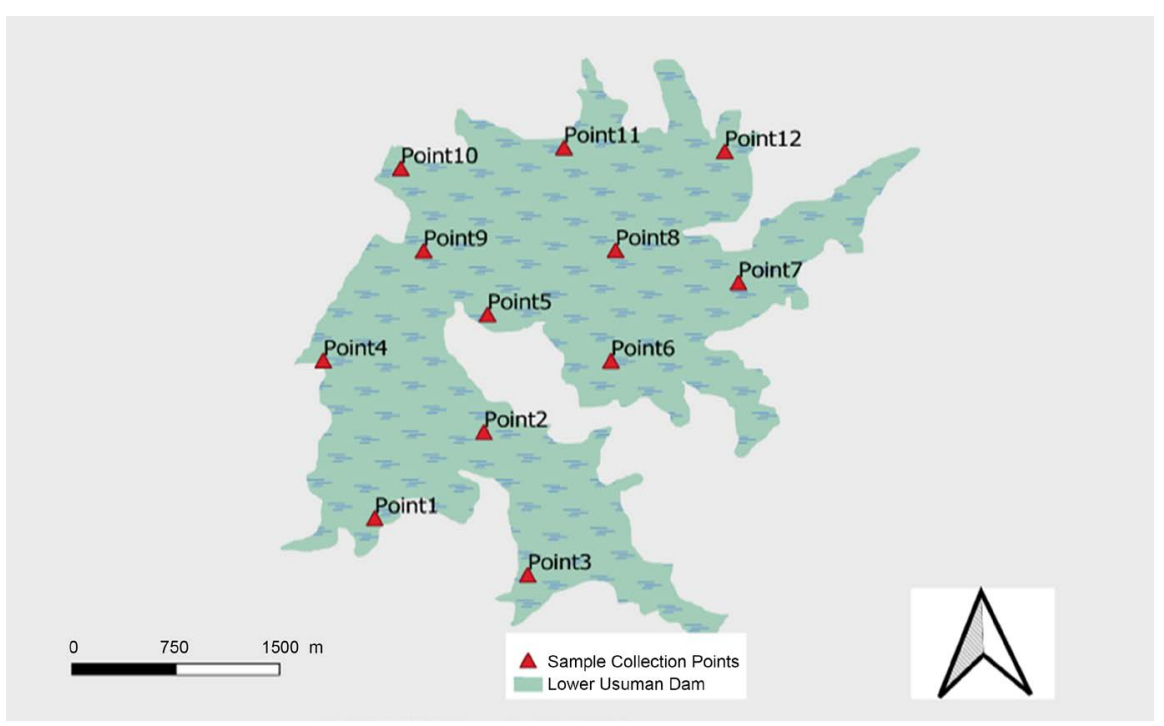

Figure 1. Lower Usuma Dam (showing sampling points). 
homogenized and kept in clean polyethylene bags. The surface layer was chosen for this study, because this layer controls the exchange of metals between sediments and waters as well as constitutes a reserve of metals to which benthic organisms are exposed (George et al., 2013). The polythene bags were then labeled to indicate sampling point and date of sampling. Samples were stored in an ice box for transportation to the laboratory. In the laboratory, the samples were kept in a freezer at $-20^{\circ} \mathrm{C}$ until they were processed for heavy metal analysis.

During digestion, the sediment samples were thawed at room temperature $\left(25^{\circ} \mathrm{C}-28^{\circ} \mathrm{C}\right)$ and put into pre-acid cleaned evaporating beakers. The sediments were then dried at a temperature of $50^{\circ} \mathrm{C}$ in an oven until a constant weight was obtained. The dried sediment samples were ground using a porcelain mortar and pestle and sieved through a $2 \mathrm{~mm}$ mesh plastic sieve.

Digestion was done using concentrated nitric acid (Analytical grade) and hydrochloric acid in the ratio 1:3 (aqua regia). $2 \mathrm{~g}$ of dry sediment was weighed using an electronic weighing balance into a $50 \mathrm{~mL}$ acid cleaned beaker. $9 \mathrm{~mL}$ of a freshly prepared mixture of $\mathrm{HNO}_{3}$ and $\mathrm{HCl}$ was added to the sediment and the mixture boiled gently over a water bath till the volume dropped to $20 \mathrm{~mL}$. The digested sample was filtered using a Whatman $0.42 \mu \mathrm{m}$ filter paper into a $50 \mathrm{~mL}$ volumetric flask and topped up to the mark with distilled-deionised water. The filtrate was analyzed for heavy metals using AAS. A blank solution was similarly prepared, using distilled water.

\subsubsection{Sampling and Digestion of Fish Samples}

Tilapia (Oreochromis spilurus niger) and African catfish (Clarias gariepinus) samples were obtained from the Lower Usuma dam by use of fishing nets that had been cast and left overnight. Fish samples were obtained from three main fishing zones in the dam twice: during the periods, July 2019 (Rainy season) and January 2020 (Dry season). The fish samples obtained were immediately kept in pre-acid cleaned polythene bags, sealed, labeled and kept in ice boxes for transportation to the laboratory. In the laboratory, the lengths and weights of the fishes were taken and the samples were kept in a deep freezer until when muscle tissues were extracted for analysis. Muscle tissues were chosen to be analyzed in this study because they are the most consumed part of the fishes. Significant levels of toxic metals, if present in the muscle tissues, will pose greater human health risk. The two fish species analyzed are the species mostly consumed from the dam all year round.

Table 1 shows the mean length and weight of the fishes.

The deep frozen fish samples for the two fish species (Oreochromis spilurus niger and Clarias gariepinus) were thawed at ambient laboratory temperature overnight. The skin of each fish sample was removed using a plastic knife (to avoid metal contamination) and this was followed by extraction of fish muscles. Fish muscles were put in pre-acid washed and oven-dried crucibles. The samples were then dried to a constant weight in an oven at $50^{\circ} \mathrm{C}$. The dried fish samples were allowed to cool in a desiccator at room temperature. 
Table 1. Mean length and weight of fish samples.

\begin{tabular}{ccc}
\hline Fish type & Mean weight $(\mathrm{kg})$ & Mean length $(\mathrm{cm})$ \\
\hline African Catfish & 0.58 & 27 \\
Tilapia & 0.33 & 12 \\
\hline
\end{tabular}

After cooling, $1 \mathrm{~g}$ of fish muscles was accurately weighed using an electronic weighing balance and transferred into a clean beaker. Dried fish samples for each fish species was digested in triplicate according to the method described in APHA (2005). To each weighed fish muscle, $18 \mathrm{~mL}$ of concentrated nitric acid was added and heated at $100^{\circ} \mathrm{C}$ on a hot plate in a fume hood chamber. A few drops of hydrogen peroxide (analytical grade) was added until there were no brown fumes.

The digested fish sample solutions were each filtered using Whatman $0.42 \mu \mathrm{m}$ filter paper in a $50 \mathrm{~mL}$ volumetric flask and topped to the mark with distilled-deionised water. The filtrate was then put into $50 \mathrm{~mL}$ pre-acid cleaned plastic bottles.

\subsubsection{Toxic Metal Assay}

Stock standard solutions containing $1000 \mathrm{mg} \cdot \mathrm{L}^{-1}$ of $\mathrm{As}, \mathrm{Cd}, \mathrm{Cr}, \mathrm{Cu}, \mathrm{Hg}, \mathrm{Ni}, \mathrm{Pb}$ and $\mathrm{Zn}$ were prepared from the metal salts (Analytical Grade) using nitric acid. Working standards for all the metals were prepared from the stock solutions by serial dilutions in distilled water. To determine the instrument signal response to changes in concentration, calibrations were done using working standard solutions of known and increasing concentrations for each analyte element of interest. The actual concentration of each toxic metal in the sediment and fish samples in $\mathrm{mg} / \mathrm{kg}$ were calculated using the formula:

$$
\begin{aligned}
& \text { Actual concentration }\left(\mathrm{mg} \cdot \mathrm{kg}^{-1}\right) \\
& =\frac{\text { Digested concentration }\left(\mathrm{mg} \cdot \mathrm{L}^{-1}\right) \times \operatorname{Volume} \operatorname{digested}(\mathrm{L})}{\text { Weight of dried sample }(\mathrm{kg})}
\end{aligned}
$$

\subsubsection{Evaluation of Ecological and Human Health Risk Indices \\ Ecological risk index}

The potential ecological risk coefficient $\left(E_{r}^{i}\right)$ of a single element and the potential ecological risk index (RI) of the multielement were computed using the following equations:

$$
\begin{aligned}
& C_{f}^{i}=C_{s}^{i} / C_{n}^{i} \\
& E_{r}^{i}=T_{r}^{i} \times C_{f}^{i} \\
& \mathrm{RI}=\sum_{i-1}^{n} E_{r}^{i}
\end{aligned}
$$

where,

$C_{f}^{i}$ is the pollution coefficient of a single element of " $i$ ";

$C_{s}^{i}$ is the measured level (sedimentary/water/fish) of heavy metal;

$C_{n}^{i}$ is the background level of heavy metal. 
The average shale background concentration of global sediments was selected as the reference baselines in this study: $C_{n}^{i}$ for $\mathrm{Cu}=45, \mathrm{Zn}=95, \mathrm{~Pb}=20, \mathrm{Cd}=$ 0.3 $, \mathrm{Cr}=90, \mathrm{Ni}=68, \mathrm{As}=13, \mathrm{Hg}=0.4$ (Turekian \& Wedepohl, 1961; Harikumar et al., 2009; Ong \& Kamaruzzaman, 2009; Jonathan et al., 2016). Average global shale values and average crustal abundance have been commonly used to provide elemental background concentrations (Uluturhan et al., 2011).

$T_{r}^{i}$ is the toxic response factor for the given element of " $i$ ", which accounts for the toxic requirement and the sensitivity requirement. The toxic response factors for $\mathrm{As}, \mathrm{Hg}, \mathrm{Pb}, \mathrm{Cd}, \mathrm{Cr}, \mathrm{Cu}, \mathrm{Zn}$ and $\mathrm{Ni}$ are 10, 40, 5, 30, 2, 5, 1 and 6 respectively (Hakanson, 1980; Xu et al., 2008; Izah et al., 2018).

Table 2 shows the grading of $E_{r}^{i}$ and RI values.

\section{Human health risk index}

1) Chronic daily intake (CDI) indices: The CDI through water and fish ingestion was calculated according to the modified equation from Chrostowski (1994):

$$
\mathrm{CDI}=\frac{\mathrm{C} \times \mathrm{DI}}{\mathrm{BW}}
$$

where, C, DI and BW represent the concentration of heavy metal $(\mathrm{mg} / \mathrm{L})$, average daily intake rate (2 L/day) and body weight (70 kg), respectively (USEPA, 2009a).

The CDI for the sediment was calculated according to the modified equations of daily intake of sedimentary toxic metals through various exposure pathways (Wang et al., 2005) shown in Table 3.

Table 2. Risk grade indices and grades of potential ecological risk of heavy metal pollution (Hakanson, 1980).

\begin{tabular}{ccccc}
\hline$E_{r}^{i}$ & Risk grade & RI value & Risk level & Risk degree \\
\hline$E_{r}^{i}<30$ & Slight & $\mathrm{RI}<40$ & $\mathrm{~A}$ & Slight \\
$30 \leq E_{r}^{i}<60$ & Medium & $40 \leq \mathrm{RI}<80$ & $\mathrm{~B}$ & Medium \\
$60 \leq E_{r}^{i}<120$ & Strong & $80 \leq \mathrm{RI}<160$ & $\mathrm{C}$ & Strong \\
$120 \leq E_{r}^{i}<240$ & Very strong & $160 \leq \mathrm{RI}<320$ & $\mathrm{D}$ & Very strong \\
$E_{r}^{i} \geq 240$ & Extremely strong & $\mathrm{RI} \geq 320$ & $\mathrm{E}$ & Extremely strong \\
\hline
\end{tabular}

Table 3. Equations of daily intake of sedimentary toxic metals through various exposure pathways (Wang et al., 2005).

\begin{tabular}{cc}
\hline Exposure Pathway & Calculation Formula \\
\hline Ingestion & $\mathrm{CDI}_{\text {Ingest }}=\frac{\mathrm{CS} \times \mathrm{IRS} \times \mathrm{EF} \times \mathrm{ED} \times \mathrm{CF}}{\mathrm{BW} \times \mathrm{AT}}$ \\
Dermal Contact & $\mathrm{CDI}_{\text {Dermal }}=\frac{\mathrm{CS} \times \mathrm{SA} \times \mathrm{AF} \times \mathrm{ABS} \times \mathrm{EF} \times \mathrm{ED}}{\mathrm{BW} \times \mathrm{AT}}$ \\
Inhalation & $\mathrm{CDI}_{\text {Inhale }}=\frac{\mathrm{CS} \times \mathrm{ET} \times \mathrm{EF} \times \mathrm{ED}}{\mathrm{PEF} \times 24 \times \mathrm{AT}}$ \\
\hline
\end{tabular}


Where:

$\mathrm{CDI}=$ chronic daily intake

$\mathrm{CS}=$ exposure-point concentration $(\mathrm{mg} / \mathrm{kg})$

$\mathrm{EF}=$ exposure frequency $=35 \mathrm{~d} / \mathrm{a}($ USEPA, 2011)

$\mathrm{ED}=$ exposure duration $=30 \mathrm{a}($ USEPA, 2011)

$\mathrm{ET}=$ exposure frequency $=24 \mathrm{~h} / \mathrm{d}(\mathrm{USDOE}, 2011)$

$\mathrm{AT}=$ averaging time for non-carcinogens $=365 \times \mathrm{ED} \mathrm{d}(\mathrm{USEPA}, 2011)$

$\mathrm{AT}=$ averaging time for carcinogens $=365 \times 70 \mathrm{~d}($ USEPA, 2002)

$\mathrm{BW}=$ body weight $=70 \mathrm{~kg}($ USEPA, 1992)

$\mathrm{SA}=$ exposed skin area $=5700 \mathrm{~cm}^{2}($ USEPA, 2011)

$\mathrm{AF}=$ adherence factor $=0.07 \mathrm{mg} \cdot \mathrm{cm}^{-2}(\mathrm{USEPA}, 2011)$

$\mathrm{ABS}=$ dermal absorption fraction $=0.03(\mathrm{As}), 0.0001$ (other metals) (USEPA, 2011)

$$
\begin{aligned}
& \mathrm{PEF}=\text { particle emission factor }=1.36 \times 10^{9} \mathrm{~m}^{3} \cdot \mathrm{kg}^{-1}(\text { USEPA, 2002) } \\
& \mathrm{CF}=\text { units conversion factor }=10^{-6} \mathrm{mg} \cdot \mathrm{kg}^{-1}(\text { USEPA, 2002) } \\
& \text { IRS }=100 \mathrm{mg} \cdot \mathrm{d}^{-1}(\text { USEPA, 2011) }
\end{aligned}
$$

2) Hazard quotient (HQ) indices: The HQ for non-carcinogenic risk was calculated by the following equation (USEPA, 2009b):

$$
\mathrm{HQ}=\frac{\mathrm{CDI}}{\mathrm{RfD}}
$$

where, the non-cancer hazard quotient (HQ) is the ratio of exposure to hazardous substances, and RFD is the chronic reference dose of the toxicant $\left(\mathrm{mg} \cdot \mathrm{kg}^{-1} \cdot \mathrm{d}^{-1}\right)$.

According to database (USEPA, 2009b), the oral toxicity reference dose values (RfD) are $\mathrm{Cr}, \mathrm{Zn}, \mathrm{Ni}, \mathrm{Cu}, \mathrm{As}, \mathrm{Cd}, \mathrm{Pb}$ and $\mathrm{Hg}$ are 0.0003, 0.3, 0.02, 0.04, 0.0005, $0.001,0.004$ and $0.0005 \mathrm{mg} / \mathrm{kg}$, respectively. The exposed population is assumed to be safe when HQ $<1$ and unsafe when HQ $\geq 1$ (Khan et al., 2008).

3) Chronic hazard index (HI)

$$
\text { Chronic Hazard Index }(\mathrm{CHI} / \mathrm{CHQ})=\sum_{i=1}^{k} \frac{\mathrm{CDI}_{k}}{\mathrm{RFD}_{k}}
$$

where, the chronic hazard index (HI) is the sum of more than one hazard quotient for multiple substances or multiple exposure pathways, $\mathrm{CDI}_{k}$ is the daily intake of a toxic metal $(k)$ and $\mathrm{RFD}_{k}$ is the chronic reference dose for the heavy metal $k$. HI values $\geq 1$ shows that there is a chance that non-carcinogenic risk may occur, and when $\mathrm{HI}<1$ the reverse applies.

4) Cancer risk: Cancer risk can be evaluated from:

$$
\text { Cancer risk }(\mathrm{CR})=\mathrm{CDI} \times \mathrm{SF}
$$

where:

Cancer risk (CR) represents the probability of an individual's lifetime health risk from carcinogens;

CDI is the chronic daily intake of carcinogens $\left(\mathrm{mg} \cdot \mathrm{kg}^{-1} \cdot \mathrm{d}^{-1}\right)$;

$\mathrm{SF}$ is the slope factor of hazardous substances $\left(\mathrm{mg} \cdot \mathrm{kg}^{-1} \cdot \mathrm{d}^{-1}\right)$.

Slope factor values for the carcinogens $\mathrm{As}, \mathrm{Ni}, \mathrm{Cd}$ and $\mathrm{Cr}$ are given as 1.5, 0.91, 15 
and 0.5 respectively (EPA, 2009). $\mathrm{Cu}, \mathrm{Zn}, \mathrm{Hg}$ and $\mathrm{Pb}$, being non-carcinogens, have no assigned value for slope factor (SF).

The cumulative cancer risk will be calculated from:

$$
\text { Total Cancer Risk }(\mathrm{TCR})=\sum_{i=1}^{k} \mathrm{CDI}_{k} \times \mathrm{SF}_{k}
$$

where:

$\mathrm{CDI}_{k}$ is the chronic daily intake $\left(\mathrm{mg} \cdot \mathrm{kg}^{-1} \cdot \mathrm{d}^{-1}\right)$ of a single toxic metal $k$;

$\mathrm{SF}_{k}$ is the slope factor for the toxic metal $k\left(\mathrm{~kg} \cdot \mathrm{d}^{-1} \cdot \mathrm{mg}^{-1}\right)$.

The acceptable or tolerable maximum limit for TCR, for regulatory purposes, is within the range of $10^{-6}-10^{-4}$ (USEPA, 2001).

\subsubsection{Statistical Analysis}

Descriptive statistics were conducted to determine the average, range, mean and standard deviations of investigated toxic metals in the water, sediment and fish samples. Pearson's correlations matrix was performed to evaluate sources of heavy metals in the studied site, the dynamics of the contamination and potential relationship among measured variables. The test of significance and one-way ANOVA were also carried out on the data.

Statistical analysis was done using IBM SPSS 23 software.

\section{Results}

\subsection{Toxic Metal Concentrations}

Tables 4-7 show the toxic metal concentrations obtained for samples from the Lower Usuma dam during the period of study.

Table 4. Toxic metal concentrations in lower Usuma dam raw water.

\begin{tabular}{ccccc}
\hline Toxic Metal & $\begin{array}{c}\text { Rainy Season } \\
\text { Conc. }(\mathrm{mg} / \mathrm{L})\end{array}$ & $\begin{array}{c}\text { Dry Season Conc. } \\
(\mathrm{mg} / \mathrm{L})\end{array}$ & $\begin{array}{c}\text { Mean Conc. } \\
(\mathrm{mg} / \mathrm{L})\end{array}$ & $\begin{array}{c}\text { WHO/NSDWQ } \\
\text { Limit }(\mathrm{mg} / \mathrm{L})\end{array}$ \\
\hline $\mathrm{As}$ & 0.0001 & 0.0008 & 0.00045 & 0.01 \\
$\mathrm{Cd}$ & -0.0021 & 0 & -0.00105 & 0.003 \\
$\mathrm{Cr}$ & -0.4918 & 0 & -0.2459 & 0.05 \\
$\mathrm{Cu}$ & 0.0014 & 0 & 0.0007 & 2.0 \\
$\mathrm{Hg}$ & -0.0002 & -0.875 & -0.4376 & 0.006 \\
$\mathrm{Ni}$ & 0.0282 & 0 & 0.0141 & 0.07 \\
$\mathrm{~Pb}$ & 0.1986 & 0 & 0.0993 & 0.01 \\
$\mathrm{Zn}$ & 0.0011 & 0.02 & 0.01055 & 2.0 \\
\hline
\end{tabular}

Table 5. Toxic metal concentration in tilapia from lower Usuma dam.

\begin{tabular}{cccccc}
\hline Toxic Metal & $\begin{array}{c}\text { Rainy Season } \\
\text { Conc. }(\mathrm{mg} / \mathrm{L})\end{array}$ & $\begin{array}{c}\text { Dry Season } \\
\text { Conc. }(\mathrm{mg} / \mathrm{L})\end{array}$ & $\begin{array}{c}\text { Mean Conc. } \\
(\mathrm{mg} / \mathrm{L})\end{array}$ & $\begin{array}{c}\text { Mean Conc. } \\
(\mathrm{mg} / \mathrm{kg})\end{array}$ & $\begin{array}{c}\text { WHO Limit } \\
(\mathrm{mg} / \mathrm{kg})\end{array}$ \\
\hline As & 0.0001 & 0.000155 & 0.000128 & 0.0063 & 0.26 \\
Cd & -0.002 & 0 & -0.001 & -0.0498 & 0.2 \\
\hline
\end{tabular}




\section{Continued}

\begin{tabular}{cccccc}
\hline $\mathrm{Cr}$ & -0.0018 & 0.02 & 0.0091 & 0.4527 & 0.5 \\
$\mathrm{Cu}$ & -0.004 & 0.0004 & -0.0018 & -0.0896 & 0.2 \\
$\mathrm{Hg}$ & -0.0001 & -0.215 & -0.10755 & -5.3507 & 0.6 \\
$\mathrm{Ni}$ & 0.0245 & 0 & 0.01225 & 0.6095 & 0.5 \\
$\mathrm{~Pb}$ & 0.2358 & 0.05 & 0.1429 & 7.1095 & 1.0 \\
$\mathrm{Zn}$ & 0.0397 & 0.414 & 0.22685 & 11.2861 & 50 \\
\hline
\end{tabular}

Table 6. Toxic metal concentration in African catfish from lower Usuma dam.

\begin{tabular}{cccccc}
\hline Toxic Metal & $\begin{array}{c}\text { Rainy Season } \\
\text { Conc. }(\mathrm{mg} / \mathrm{L})\end{array}$ & $\begin{array}{c}\text { Dry Season } \\
\text { Conc. }(\mathrm{mg} / \mathrm{L})\end{array}$ & $\begin{array}{c}\text { Mean Conc. } \\
(\mathrm{mg} / \mathrm{L})\end{array}$ & $\begin{array}{c}\text { Mean Conc. } \\
(\mathrm{mg} / \mathrm{kg})\end{array}$ & $\begin{array}{c}\text { WHO Limit } \\
(\mathrm{mg} / \mathrm{kg})\end{array}$ \\
\hline $\mathrm{As}$ & 0.0001 & 0.00013 & 0.000115 & 0.00571 & 0.26 \\
$\mathrm{Cd}$ & -0.0014 & 0 & -0.0007 & -0.03476 & 0.2 \\
$\mathrm{Cr}$ & -0.1875 & 0.02 & -0.08375 & -4.15839 & 0.5 \\
$\mathrm{Cu}$ & -0.0004 & 0.0007 & 0.00015 & 0.007448 & 0.2 \\
$\mathrm{Hg}$ & 0.0001 & -0.255 & -0.12745 & -6.3282 & 0.6 \\
$\mathrm{Ni}$ & 0.0206 & 0.01 & 0.0153 & 0.759682 & 0.5 \\
$\mathrm{~Pb}$ & 0.0815 & 0.07 & 0.07575 & 3.761172 & 1.0 \\
$\mathrm{Zn}$ & 0.0308 & 0.11555 & 0.073175 & 3.633317 & 50 \\
\hline
\end{tabular}

Table 7. Toxic metal concentration in sediment from lower Usuma dam.

\begin{tabular}{cccccc}
\hline Toxic Metal & $\begin{array}{c}\text { Rainy Season } \\
\text { Conc. }(\mathrm{mg} / \mathrm{L})\end{array}$ & $\begin{array}{c}\text { Dry Season } \\
\text { Conc. }(\mathrm{mg} / \mathrm{L})\end{array}$ & $\begin{array}{c}\text { Mean Conc. } \\
(\mathrm{mg} / \mathrm{L})\end{array}$ & $\begin{array}{c}\text { Mean Conc. } \\
(\mathrm{mg} / \mathrm{kg})\end{array}$ & $\begin{array}{c}\text { ICPR Limit } \\
(\mathrm{mg} / \mathrm{kg})\end{array}$ \\
\hline $\mathrm{As}$ & 0.0013 & 0.00145 & 0.001375 & 0.034 & 1.0 \\
$\mathrm{Cd}$ & -0.0018 & 0.01 & 0.0041 & 0.102 & 0.1 \\
$\mathrm{Cr}$ & -0.0015 & 0.15 & 0.07425 & 1.855 & 30.0 \\
$\mathrm{Cu}$ & 0.0004 & 0.017 & 0.0087 & 0.217 & 25.0 \\
$\mathrm{Hg}$ & 0.0001 & -16.645 & -8.32245 & -207.957 & 0.5 \\
$\mathrm{Ni}$ & 0.0304 & -0.1 & -0.0348 & -0.870 & 50.0 \\
$\mathrm{~Pb}$ & 0.1297 & 0.175 & 0.15235 & 3.807 & 40.0 \\
$\mathrm{Zn}$ & 0.0022 & 2.49625 & 1.249225 & 31.215 & 90.0 \\
\hline
\end{tabular}

\subsection{Ecological Risk Index}

Values for contamination factor $\left(C_{f}^{i}\right)$ and Ecological risk index $\left(E_{r}^{i}\right)$ determined are shown in Tables 8-11.

\subsection{Human Health Risk Index}

The Hazard Quotient (HQ) and Cancer Risk (CR) index values obtained are shown in Tables 12-17. 
Table 8. Ecological risk index for toxic metals in water.

\begin{tabular}{ccccccc}
\hline Toxic Metal & Mean $C_{s}^{i}$ & $C_{n}^{i}$ & $C_{f}^{i}$ & $T_{r}^{i}$ & $E_{r}^{i}$ & Risk Grade \\
\hline $\mathrm{As}$ & 0.00045 & 13 & $3.46 \times 10^{-05}$ & 10 & $3.46 \times 10^{-04}$ & Slight \\
$\mathrm{Cd}$ & -0.00105 & 0.3 & $-3.50 \times 10^{-03}$ & 30 & $-1.05 \times 10^{-01}$ & Slight \\
$\mathrm{Cr}$ & -0.2459 & 90 & $-2.73 \times 10^{-03}$ & 2 & $-5.46 \times 10^{-03}$ & Slight \\
$\mathrm{Cu}$ & 0.0007 & 45 & $1.56 \times 10^{-05}$ & 5 & $7.78 \times 10^{-05}$ & Slight \\
$\mathrm{Hg}$ & -0.4376 & 0.4 & -1.09 & 40 & $-4.38 \times 10^{+01}$ & Slight \\
$\mathrm{Ni}$ & 0.0141 & 68 & $2.07 \times 10^{-04}$ & 6 & $1.24 \times 10^{-03}$ & Slight \\
$\mathrm{Pb}$ & 0.0993 & 20 & $4.97 \times 10^{-03}$ & 5 & $2.48 \times 10^{-02}$ & Slight \\
$\mathrm{Zn}$ & 0.01055 & 95 & $1.11 \times 10^{-04}$ & 1 & $1.11 \times 10^{-04}$ & Slight \\
\hline
\end{tabular}

$\mathrm{RI}=\sum_{i-1}^{n} E_{r}^{i}=-43.844$; Slight Ecological Risk.

Table 9. Ecological risk index for toxic metals in catfish.

\begin{tabular}{ccccccc}
\hline Toxic Metal & Mean $C_{s}^{i}$ & $C_{n}^{i}$ & $C_{f}^{i}$ & $T_{r}^{i}$ & $E_{r}^{i}$ & Risk Grade \\
\hline $\mathrm{As}$ & 0.00571 & 13 & $4.39 \times 10^{-04}$ & 10 & 0.057 & Slight \\
$\mathrm{Cd}$ & -0.0348 & 0.3 & $-1.16 \times 10^{-01}$ & 30 & -1.043 & Slight \\
$\mathrm{Cr}$ & -4.1584 & 90 & $-4.62 \times 10^{-02}$ & 2 & -8.317 & Slight \\
$\mathrm{Cu}$ & 0.00745 & 45 & $1.66 \times 10^{-04}$ & 5 & 0.037 & Slight \\
$\mathrm{Hg}$ & -6.3282 & 0.4 & $-1.58 \times 10^{-01}$ & 40 & -253.128 & Slight \\
$\mathrm{Ni}$ & 0.75968 & 68 & $1.12 \times 10^{-02}$ & 6 & 4.558 & Slight \\
$\mathrm{Pb}$ & 3.76117 & 20 & $1.88 \times 10^{-01}$ & 5 & 18.806 & Slight \\
$\mathrm{Zn}$ & 3.63332 & 95 & $3.82 \times 10^{-02}$ & 1 & 3.633 & Slight \\
\hline
\end{tabular}

$\mathrm{RI}=\sum_{i-1}^{n} E_{r}^{i}=-235.396 ;$ Slight Ecological Risk.

Table 10. Ecological risk index for toxic metals in tilapia.

\begin{tabular}{ccccccc}
\hline Toxic Metal & Mean $C_{s}^{i}$ & $C_{n}^{i}$ & $C_{f}^{i}$ & $T_{r}^{i}$ & $E_{r}^{i}$ & Risk Grade \\
\hline $\mathrm{As}$ & 0.0063 & 13 & $4.85 \times 10^{-04}$ & 10 & 0.063 & Slight \\
$\mathrm{Cd}$ & -0.0498 & 0.3 & $-1.66 \times 10^{-01}$ & 30 & -1.49 & Slight \\
$\mathrm{Cr}$ & 0.4527 & 90 & $5.03 \times 10^{-03}$ & 2 & 0.91 & Slight \\
$\mathrm{Cu}$ & -0.0896 & 45 & $-1.99 \times 10^{-03}$ & 5 & -0.45 & Slight \\
$\mathrm{Hg}$ & -5.3507 & 0.4 & $-1.34 \times 10^{1}$ & 40 & -214.03 & Slight \\
$\mathrm{Ni}$ & 0.6095 & 68 & $8.96 \times 10^{-03}$ & 6 & 3.66 & Slight \\
$\mathrm{Pb}$ & 7.1095 & 20 & $3.55 \times 10^{-01}$ & 5 & 35.55 & Medium \\
$\mathrm{Zn}$ & 11.2861 & 95 & $1.19 \times 10^{-01}$ & 1 & 11.29 & Slight \\
\hline
\end{tabular}

$\mathrm{RI}=\sum_{i-1}^{n} E_{r}^{i}=-164.51$; Slight Ecological Risk.

Table 11. Ecological risk index for toxic metals in sediment.

\begin{tabular}{ccccccc}
\hline Toxic Metal & Mean $C_{s}^{i}$ & $C_{n}^{i}$ & $C_{f}^{i}$ & $T_{r}^{i}$ & $E_{r}^{i}$ & Risk Grade \\
\hline $\mathrm{As}$ & 0.197 & 13 & $1.52 \times 10^{-02}$ & 10 & 1.97 & Slight \\
$\mathrm{Cd}$ & 0.102 & 0.3 & $3.40 \times 10^{-01}$ & 30 & 3.06 & Slight \\
$\mathrm{Cr}$ & 1.855 & 90 & $2.06 \times 10^{-02}$ & 2 & 3.71 & Slight \\
$\mathrm{Cu}$ & 2.129 & 45 & $4.73 \times 10^{-02}$ & 5 & 10.645 & Slight \\
$\mathrm{Hg}$ & -207.957 & 0.4 & $-5.20 \times 10^{02}$ & 40 & -8318.28 & Slight \\
\hline
\end{tabular}


R. Wuana et al.

Continued

\begin{tabular}{ccccccc}
\hline $\mathrm{Ni}$ & -0.870 & 68 & $-1.28 \times 10^{-02}$ & 6 & -5.22 & Slight \\
$\mathrm{Pb}$ & 3.807 & 20 & $1.90 \times 10^{-01}$ & 5 & 19.035 & Slight \\
$\mathrm{Zn}$ & 31.215 & 95 & $3.29 \times 10^{-01}$ & 1 & 31.215 & Medium \\
\hline
\end{tabular}

$\mathrm{RI}=\sum_{i-1}^{n} E_{r}^{i}=-8.3 \times 10^{3} ;$ Slight Ecological Risk.

Table 12. Human health risk index from consumption of toxic metals in LUD water.

\begin{tabular}{cccccccc}
\hline $\begin{array}{c}\text { Toxic } \\
\text { Metal }\end{array}$ & $\begin{array}{c}\text { MeanConc. } \\
(\mathrm{mg} / \mathrm{L})\end{array}$ & CDI & RfD & HQ & Safety & SF & CR \\
\hline $\mathrm{As}$ & -0.0021 & $1.29 \times 10^{-05}$ & 0.0005 & 0.0257 & Safe & 1.5 & $1.93 \times 10^{-05}$ \\
$\mathrm{Cd}$ & -0.4918 & $-3.00 \times 10^{-05}$ & 0.001 & -0.03 & Safe & 15 & $-4.50 \times 10^{-04}$ \\
$\mathrm{Cr}$ & 0.0014 & $-7.03 \times 10^{-03}$ & 0.0003 & -23.419 & Safe & 0.5 & $-3.51 \times 10^{-03}$ \\
$\mathrm{Cu}$ & -0.0002 & $2.00 \times 10^{-05}$ & 0.04 & 0.0005 & Safe & - & \\
$\mathrm{Hg}$ & 0.0282 & $-1.25 \times 10^{-02}$ & 0.0005 & -25.0057 & Safe & - & \\
$\mathrm{Ni}$ & 0.1986 & $4.03 \times 10^{-04}$ & 0.02 & 0.020143 & Safe & 0.91 & $3.67 \times 10^{-04}$ \\
$\mathrm{~Pb}$ & 0.0011 & $2.84 \times 10^{-03}$ & 0.004 & 0.709286 & Safe & - & \\
$\mathrm{Zn}$ & -0.0021 & $3.01 \times 10^{-04}$ & 0.3 & 0.001005 & Safe & - & \\
\hline
\end{tabular}

Chronic hazard index $=\Sigma H Q=-26.60$; Total cancer risk $=\Sigma C R=-3.58 \times 10^{-3}$.

Table 13. Human health risk index from consumption of toxic metals in LUD catfish.

\begin{tabular}{cccccccc}
\hline $\begin{array}{c}\text { Toxic } \\
\text { Metal }\end{array}$ & $\begin{array}{c}\text { MeanConc. } \\
(\mathbf{m g} / \mathbf{k g})\end{array}$ & CDI & RfD & HQ & Safety & SF & CR \\
\hline $\mathrm{As}$ & 0.00571 & $1.63 \times 10^{-04}$ & 0.0003 & 0.54381 & Safe & 1.5 & $1.09 \times 10^{-04}$ \\
$\mathrm{Cd}$ & -0.03476 & $-9.93 \times 10^{-04}$ & 0.001 & -0.99314 & Safe & 15 & $-6.62 \times 10^{-05}$ \\
$\mathrm{Cr}$ & -4.15839 & $-1.19 \times 10^{-01}$ & 0.003 & -39.6037 & Safe & 0.5 & $-2.38 \times 10^{-01}$ \\
$\mathrm{Cu}$ & 0.007448 & $2.13 \times 10^{-04}$ & 0.04 & 0.00532 & Safe & - & - \\
$\mathrm{Hg}$ & -6.3282 & $-1.81 \times 10^{-01}$ & 0.0005 & -361.611 & Safe & - & - \\
$\mathrm{Ni}$ & 0.759682 & $2.17 \times 10^{-02}$ & 0.02 & 1.08526 & Unsafe & 0.91 & $2.39 \times 10^{-02}$ \\
$\mathrm{~Pb}$ & 3.761172 & $1.07 \times 10^{-01}$ & 0.004 & 26.86551 & Unsafe & - & - \\
$\mathrm{Zn}$ & 3.633317 & $1.04 \times 10^{-01}$ & 0.3 & 0.34603 & Safe & - & - \\
\hline
\end{tabular}

Chronic hazard index $=\Sigma \mathrm{HQ}=-373.362$; Total cancer risk $=\Sigma \mathrm{CR}=-2.14 \times 10^{-01}$.

Table 14. Human health risk index table from consumption of toxic metals in LUD tilapia.

\begin{tabular}{cccccccc}
\hline $\begin{array}{c}\text { Toxic } \\
\text { Metal }\end{array}$ & $\begin{array}{c}\text { MeanConc. } \\
(\mathrm{mg} / \mathrm{kg})\end{array}$ & CDI & RfD & HQ & Safety & SF & CR \\
\hline $\mathrm{As}$ & 0.0063 & $1.80 \times 10^{-04}$ & 0.0003 & 0.6 & Safe & 1.5 & $1.20 \times 10^{-04}$ \\
$\mathrm{Cd}$ & -0.0498 & $-1.42 \times 10^{-03}$ & 0.001 & -1.42286 & Safe & 15 & $-9.49 \times 10^{-05}$ \\
$\mathrm{Cr}$ & 0.4527 & $1.29 \times 10^{-02}$ & 0.003 & 4.311429 & Unsafe & 0.5 & $2.59 \times 10^{-02}$ \\
$\mathrm{Cu}$ & -0.0896 & $-2.56 \times 10^{-03}$ & 0.04 & -0.064 & Safe & - & - \\
$\mathrm{Hg}$ & -5.3507 & $-1.53 \times 10^{-01}$ & 0.0005 & -305.754 & Safe & - & - \\
$\mathrm{Ni}$ & 0.6095 & $1.74 \times 10^{-02}$ & 0.02 & 0.870714 & Safe & 0.91 & $1.91 \times 10^{-02}$ \\
$\mathrm{~Pb}$ & 7.1095 & $2.03 \times 10^{-01}$ & 0.004 & 50.78214 & Unsafe & - & - \\
$\mathrm{Zn}$ & 11.2861 & $3.22 \times 10^{-01}$ & 0.3 & 1.074867 & Unsafe & - & - \\
\hline
\end{tabular}

Chronic hazard index $=\Sigma \mathrm{HQ}=-249.602$; Total cancer risk $=\Sigma \mathrm{CR}=4.50 \times 10^{-2}$. 
Table 15. Human health risk index table from ingestion of toxic metals in LUD sediment.

\begin{tabular}{cccccccc}
\hline $\begin{array}{c}\text { Toxic } \\
\text { Metal }\end{array}$ & $\begin{array}{c}\text { MeanConc. } \\
(\mathrm{mg} / \mathrm{kg})\end{array}$ & CDI $_{\text {ingestion }}$ & $\mathrm{RfD}$ & $\mathrm{HQ}$ & Safety & $\mathrm{SF}$ & $\mathrm{CR}$ \\
\hline $\mathrm{As}$ & 0.03 & $2.02 \times 10^{-09}$ & 0.0003 & $4.03 \times 10^{-06}$ & Safe & 1.5 & $3.03 \times 10^{-09}$ \\
$\mathrm{Cd}$ & 0.10 & $6.01 \times 10^{-09}$ & 0.001 & $6.01 \times 10^{-06}$ & Safe & 15 & $9.02 \times 10^{-08}$ \\
$\mathrm{Cr}$ & 1.86 & $1.09 \times 10^{-07}$ & 0.003 & $3.63 \times 10^{-04}$ & Safe & 0.5 & $5.45 \times 10^{-08}$ \\
$\mathrm{Cu}$ & 0.22 & $2.98 \times 10^{-08}$ & 0.04 & $7.45 \times 10^{-07}$ & Safe & - & - \\
$\mathrm{Hg}$ & -207.96 & $-2.85 \times 10^{-05}$ & 0.0005 & $-5.70 \times 10^{-02}$ & Safe & - & - \\
$\mathrm{Ni}$ & -0.87 & $-5.11 \times 10^{-08}$ & 0.02 & $-2.55 \times 10^{-06}$ & Safe & 0.91 & $-4.65 \times 10^{-08}$ \\
$\mathrm{~Pb}$ & 3.81 & $5.21 \times 10^{-07}$ & 0.004 & $1.30 \times 10^{-04}$ & Safe & - & - \\
$\mathrm{Zn}$ & 31.22 & $4.28 \times 10^{-06}$ & 0.3 & $1.43 \times 10^{-05}$ & Safe & - & - \\
\hline
\end{tabular}

Chronic hazard index $=\Sigma \mathrm{HQ}=-5.64 \times 10^{-02}$; Total cancer risk $=\Sigma \mathrm{CR}=1.01 \times 10^{-07}$.

Table 16. Human health risk index table from inhalation of toxic metals in LUD sediment.

\begin{tabular}{cccccccc}
\hline $\begin{array}{c}\text { Toxic } \\
\text { Metal }\end{array}$ & $\begin{array}{c}\text { MeanConc. } \\
(\mathbf{m g} / \mathrm{kg})\end{array}$ & $\mathrm{CDI}_{\text {inhalation }}$ & RfD & HQ & Safety & SF & CR \\
\hline $\mathrm{As}$ & 0.03 & $1.04 \times 10^{-12}$ & 0.0003 & $2.08 \times 10^{-09}$ & Safe & 1.5 & $1.56 \times 10^{-12}$ \\
$\mathrm{Cd}$ & 0.10 & $3.09 \times 10^{-12}$ & 0.001 & $3.09 \times 10^{-09}$ & Safe & 15 & $4.64 \times 10^{-11}$ \\
$\mathrm{Cr}$ & 1.86 & $5.60 \times 10^{-11}$ & 0.003 & $1.87 \times 10^{-07}$ & Safe & 0.5 & $2.80 \times 10^{-11}$ \\
$\mathrm{Cu}$ & 0.22 & $1.53 \times 10^{-11}$ & 0.04 & $3.83 \times 10^{-10}$ & Safe & - & - \\
$\mathrm{Hg}$ & -207.96 & $-1.47 \times 10^{-08}$ & 0.0005 & $-2.93 \times 10^{-05}$ & Safe & - & - \\
$\mathrm{Ni}$ & -0.87 & $-2.63 \times 10^{-11}$ & 0.02 & $-1.31 \times 10^{-09}$ & Safe & 0.91 & $-2.39 \times 10^{-11}$ \\
$\mathrm{~Pb}$ & 3.81 & $2.68 \times 10^{-10}$ & 0.004 & $6.71 \times 10^{-08}$ & Safe & - & - \\
$\mathrm{Zn}$ & 31.22 & $2.20 \times 10^{-09}$ & 0.3 & $7.34 \times 10^{-09}$ & Safe & - & - \\
\hline
\end{tabular}

Chronic hazard index $=\Sigma \mathrm{HQ}=-2.91 \times 10^{-05}$; Total cancer risk $=\Sigma \mathrm{CR}=5.21 \times 10^{-11}$.

Table 17. Human health risk index table from dermal contact with toxic metals in LUD sediment.

\begin{tabular}{cccccccc}
\hline $\begin{array}{c}\text { Toxic } \\
\text { Metal }\end{array}$ & $\begin{array}{c}\text { Mean Conc. } \\
(\mathrm{mg} / \mathrm{kg})\end{array}$ & CDI $_{\text {dermal }}$ & RfD & HQ & Safety & SF & CR \\
\hline $\mathrm{As}$ & 0.03 & 0.0881 & 0.0003 & -176.26 & Safe & 1.5 & $1.32 \times 10^{-01}$ \\
$\mathrm{Cd}$ & 0.10 & 0.0009 & 0.001 & 0.88 & Safe & 15 & $1.31 \times 10^{-02}$ \\
$\mathrm{Cr}$ & 1.86 & 0.0159 & 0.003 & -52.88 & Safe & 0.5 & $7.93 \times 10^{-03}$ \\
$\mathrm{Cu}$ & 0.22 & 0.0043 & 0.04 & 0.11 & Unsafe & - & - \\
$\mathrm{Hg}$ & -207.96 & -4.1487 & 0.0005 & -8297.50 & Safe & - & - \\
$\mathrm{Ni}$ & -0.87 & -0.0074 & 0.02 & -0.37 & Safe & 0.91 & $-6.77 \times 10^{-03}$ \\
$\mathrm{~Pb}$ & 3.81 & 0.0759 & 0.004 & 18.99 & Unsafe & - & - \\
$\mathrm{Zn}$ & 31.22 & 0.0759 & 0.3 & 0.25 & Safe & - & - \\
\hline
\end{tabular}

Chronic hazard index $=\Sigma \mathrm{HQ}=-8048.51$; Total cancer risk $=\Sigma \mathrm{CR}=1.46 \times 10^{-01}$.

\subsection{Statistical Analysis}

Tables 18-30 show the results of t-test, ANOVA and Pearson correlation generated from the data in Section 3.1. 
Table 18. Rainy Season t-test result of raw water and toxic metals concentration in tilapia.

\begin{tabular}{|c|c|c|c|c|c|c|}
\hline & \multirow[t]{2}{*}{$\mathrm{T}$} & \multirow[t]{2}{*}{ Df } & \multirow[t]{2}{*}{ Sig. (2-tailed) } & \multirow[t]{2}{*}{ Mean Difference } & \multicolumn{2}{|c|}{$\begin{array}{c}\text { 95\% Confidence Interval of the } \\
\text { Difference }\end{array}$} \\
\hline & & & & & Lower & Upper \\
\hline Raw W & -0.47 & 7 & 0.65 & -0.03 & -0.20 & 0.13 \\
\hline Tilapia Fish & 1.26 & 7 & 0.25 & 0.03 & -0.03 & 0.11 \\
\hline
\end{tabular}

Table 19. ANOVA result toxic metal concentration in tilapia from lower Usuma dam during rainy season.

\begin{tabular}{cccccc}
\hline & Sum of Squares & Df & Mean Square & F & Sig. \\
\hline Between Groups & 0.05 & 5 & 0.009 & 53.80 & 0.02 \\
Within Groups & 0.00 & 2 & 0.000 & & \\
Total & 0.05 & 7 & & \\
\hline
\end{tabular}

Table 20. Dry season t-test result of raw water and toxic metals concentration in tilapia.

\begin{tabular}{|c|c|c|c|c|c|c|}
\hline & \multirow[t]{2}{*}{$\mathrm{T}$} & \multirow[t]{2}{*}{ Df } & \multirow[t]{2}{*}{ Sig. (2-tailed) } & \multirow[t]{2}{*}{ Mean Difference } & \multicolumn{2}{|c|}{$\begin{array}{l}\text { 95\% Confidence Interval of the } \\
\text { Difference }\end{array}$} \\
\hline & & & & & Lower & Upper \\
\hline Raw W & -0.97 & 7 & 0.36 & -0.11 & -0.37 & 0.15 \\
\hline Tilapia Fish & 0.55 & 7 & 0.60 & 0.03 & -0.11 & 0.18 \\
\hline
\end{tabular}

Table 21. ANOVA result toxic metal concentration in tilapia from lower Usuma dam during rainy season.

\begin{tabular}{cccccc}
\hline & Sum of Squares & Df & Mean Square & F & Sig. \\
\hline Between Groups & 0.211 & 5 & 0.042 & 422.31 & 0.002 \\
Within Groups & 0.000 & 2 & 0.000 & & \\
Total & 0.211 & 7 & & \\
\hline
\end{tabular}

Table 22. Correlation between rainy and dry season of metal concentration in tilapia from lower Usuma dam.

\begin{tabular}{|c|c|c|c|c|c|}
\hline & & T F Rainy S & T F Dry S & Raw W R S & Raw W DS \\
\hline & Pearson Correlation & 1 & 0.18 & 0.51 & 0.18 \\
\hline \multirow[t]{3}{*}{ T F Rainy S } & Sig. (2-tailed) & & 0.66 & 0.20 & 0.67 \\
\hline & $\mathrm{N}$ & 8 & 8 & 8 & 8 \\
\hline & Pearson Correlation & 0.18 & 1 & 0.04 & 0.60 \\
\hline \multirow[t]{3}{*}{ T F Dry S } & Sig. (2-tailed) & 0.66 & & 0.93 & 0.120 \\
\hline & $\mathrm{N}$ & 8 & 8 & 8 & 8 \\
\hline & Pearson Correlation & 0.512 & 0.040 & 1 & -0.065 \\
\hline \multirow[t]{3}{*}{ Raw W R S } & Sig. (2-tailed) & 0.195 & 0.926 & & 0.878 \\
\hline & $\mathrm{N}$ & 8 & 8 & 8 & 8 \\
\hline & Pearson Correlation & 0.180 & 0.596 & -0.065 & 1 \\
\hline \multirow[t]{2}{*}{ Raw W DS } & Sig. (2-tailed) & 0.670 & 0.119 & 0.878 & \\
\hline & $\mathrm{N}$ & 8 & 8 & 8 & 8 \\
\hline
\end{tabular}


Table 23. T-test result of toxic metal concentration in African catfish from lower Usuma dam.

\begin{tabular}{ccccccc}
\hline & & & & & \multicolumn{2}{c}{$95 \%$ Confidence Interval of the } \\
& T & Df & Sig. (2-tailed) & $\begin{array}{c}\text { Mean } \\
\text { Difference }\end{array}$ & \multicolumn{2}{c}{ Difference } \\
\cline { 5 - 7 } & & & & & Lower & Upper \\
\hline Raw W R S & -0.47 & 7 & 0.65 & -0.03 & -0.20 & 0.13 \\
C F Rainy S & -0.25 & 7 & 0.81 & -0.01 & -0.07 & 0.060 \\
\hline
\end{tabular}

Table 24. Rainy season ANOVA result of raw water and toxic metals concentration in Tilapia.

\begin{tabular}{cccccc}
\hline & Sum of Squares & df & Mean Square & F & Sig. \\
\hline Between Groups & 0.02 & 5 & 0.00 & 0.39 & 0.83 \\
Within Groups & 0.02 & 2 & 0.01 & \\
Total & 0.04 & 7 & & \\
\hline
\end{tabular}

Table 25. T-test result of toxic metal concentration in African catfish from lower Usuma Dam during dry season.

\begin{tabular}{|c|c|c|c|c|c|c|}
\hline & \multirow[t]{2}{*}{$\mathrm{T}$} & \multirow[t]{2}{*}{ Df } & \multirow[t]{2}{*}{ Sig. (2-tailed) } & \multirow{2}{*}{$\begin{array}{c}\text { Mean } \\
\text { Difference }\end{array}$} & \multicolumn{2}{|c|}{$\begin{array}{c}95 \% \text { Confidence } \\
\text { Interval of the Difference }\end{array}$} \\
\hline & & & & & Lower & Upper \\
\hline Raw W DS & -0.97 & 7 & 0.36 & -0.11 & -0.37 & 0.15 \\
\hline C F Dry S & -0.13 & 7 & 0.90 & -0.01 & -0.10 & 0.09 \\
\hline
\end{tabular}

Table 26. Dry season ANOVA result of raw water and toxic metals concentration in catfish.

\begin{tabular}{cccccc}
\hline & Sum of Squares & Df & Mean Square & F & Sig. \\
\hline Between Groups & 0.08 & 5 & 0.01 & 665.07 & 0.00 \\
Within Groups & 0.00 & 2 & 0.00 & & \\
Total & 0.08 & 7 & & \\
\hline
\end{tabular}

Table 27. Correlation between rainy and dry season of metal concentration in catfish from lower Usuma dam.

\begin{tabular}{cccccc}
\hline & & C F Rainy S & C F Dry S & Raw W R S & Raw W DS \\
\hline \multirow{3}{*}{ C F Rainy S } & Pearson Correlation & 1 & 0.09 & 0.99 & -0.03 \\
& Sig. (2-tailed) & & 0.83 & 0.00 & 0.94 \\
& N & 8 & 8 & 8 & 8 \\
\hline \multirow{3}{*}{ C F Dry S } & Pearson Correlation & 0.09 & 1 & 0.02 & 0.93 \\
& Sig. (2-tailed) & 0.83 & & 0.96 & 0.00 \\
& N & 8 & 8 & 8 & 8 \\
\hline \multirow{3}{*}{ Raw W R S } & Pearson Correlation & 0.99 & 0.02 & 1 & -0.07 \\
& Sig. (2-tailed) & 0.00 & 0.96 & & 0.88 \\
& N & 8 & 8 & 8 & 8 \\
\hline
\end{tabular}




\section{Continued}

\begin{tabular}{cccccc}
\hline & Pearson Correlation & -0.03 & 0.9 & -0.07 & 1 \\
\multirow{2}{*}{ Raw W DS } & Sig. (2-tailed) & 0.94 & 0.00 & 0.88 & \\
& $\mathrm{~N}$ & 8 & 8 & 8 & 8 \\
\hline
\end{tabular}

Table 28. ANOVA result of toxic metal concentration in sediment from lower Usuma dam.

\begin{tabular}{cccccc}
\hline & Sum of Squares & Df & Mean Square & F & Sig. \\
\hline Between Groups & 0.015 & 7 & 0.002 & 0.00 & 0.00 \\
Within Groups & 0.000 & 0 &. & & \\
Total & 0.015 & 7 & & & \\
\hline
\end{tabular}

Table 29. ANOVA result of toxic metal concentration in sediment from lower Usuma dam.

\begin{tabular}{cccccc}
\hline & Sum of Squares & df & Mean Square & F & Sig. \\
\hline Between Groups & 259.216 & 7 & 37.031 & 0.00 & 0.00 \\
Within Groups & 0.000 & 0 &. & & \\
Total & 259.216 & 7 & & &
\end{tabular}

Table 30. Correlation between rainy and dry season of metal concentration in sediment.

\begin{tabular}{|c|c|c|c|c|c|c|c|c|c|}
\hline & & $\begin{array}{l}\text { Raw W } \\
\text { Rainy S }\end{array}$ & $\begin{array}{c}\text { Raw W } \\
\text { Dry S }\end{array}$ & Sed. Rainy S & $\begin{array}{c}\text { Sed } \\
\text { Dry S }\end{array}$ & T F Rainy S & T F Dry S & $\begin{array}{c}\text { C F } \\
\text { Rainy S }\end{array}$ & $\begin{array}{l}\text { C F } \\
\text { Dry S }\end{array}$ \\
\hline & Pearson Correlation & 1 & -0.065 & 0.518 & -0.059 & 0.512 & 0.040 & 0.991 & 0.021 \\
\hline \multirow[t]{3}{*}{ Raw W R S } & Sig. (2-tailed) & & 0.878 & 0.189 & 0.890 & 0.195 & 0.926 & 0.000 & 0.960 \\
\hline & $\mathrm{N}$ & 8 & 8 & 8 & 8 & 8 & 8 & 8 & 8 \\
\hline & Pearson Correlation & -0.065 & 1 & 0.172 & 0.993 & 0.180 & 0.596 & -0.032 & 0.932 \\
\hline \multirow[t]{3}{*}{ Raw W DS } & Sig. (2-tailed) & 0.878 & & 0.684 & 0.000 & 0.670 & 0.119 & 0.940 & 0.001 \\
\hline & $\mathrm{N}$ & 8 & 8 & 8 & 8 & 8 & 8 & 8 & 8 \\
\hline & Pearson Correlation & 0.518 & 0.172 & 1 & 0.155 & 0.977 & 0.035 & 0.508 & 0.297 \\
\hline \multirow[t]{3}{*}{ Sed R S } & Sig. (2-tailed) & 0.189 & 0.684 & & 0.714 & 0.000 & 0.934 & 0.199 & 0.475 \\
\hline & $\mathrm{N}$ & 8 & 8 & 8 & 8 & 8 & 8 & 8 & 8 \\
\hline & Pearson Correlation & -0.059 & 0.993 & 0.155 & 1 & 0.185 & 0.688 & -0.012 & 0.962 \\
\hline \multirow[t]{3}{*}{ Sed D S } & Sig. (2-tailed) & 0.890 & 0.000 & 0.714 & & 0.661 & 0.059 & 0.978 & 0.000 \\
\hline & $\mathrm{N}$ & 8 & 8 & 8 & 8 & 8 & 8 & 8 & 8 \\
\hline & Pearson Correlation & 0.512 & 0.180 & 0.977 & 0.185 & 1 & 0.184 & 0.520 & 0.362 \\
\hline \multirow[t]{3}{*}{ T F RAiny $\mathrm{S}$} & Sig. (2-tailed) & 0.195 & 0.670 & 0.000 & 0.661 & & 0.663 & 0.187 & 0.378 \\
\hline & $\mathrm{N}$ & 8 & 8 & 8 & 8 & 8 & 8 & 8 & 8 \\
\hline & Pearson Correlation & 0.040 & 0.596 & 0.035 & 0.688 & 0.184 & 1 & 0.157 & 0.811 \\
\hline \multirow[t]{3}{*}{ T F Dry S } & Sig. (2-tailed) & 0.926 & 0.119 & 0.934 & 0.059 & 0.663 & & 0.710 & 0.015 \\
\hline & $\mathrm{N}$ & 8 & 8 & 8 & 8 & 8 & 8 & 8 & 8 \\
\hline & Pearson Correlation & 0.991 & -0.032 & 0.508 & -0.012 & 0.520 & 0.157 & 1 & 0.091 \\
\hline \multirow[t]{2}{*}{ C F Rainy S } & Sig. (2-tailed) & 0.000 & 0.940 & 0.199 & 0.978 & 0.187 & 0.710 & & 0.831 \\
\hline & $\mathrm{N}$ & 8 & 8 & 8 & 8 & 8 & 8 & 8 & 8 \\
\hline
\end{tabular}




\begin{tabular}{ccccccccccc} 
Continued & \multicolumn{10}{l}{} \\
\hline & Pearson Correlation & 0.021 & 0.932 & 0.297 & 0.962 & 0.362 & 0.811 & 0.091 & 1 \\
C F Dry S & Sig. (2-tailed) & 0.960 & 0.001 & 0.475 & 0.000 & 0.378 & 0.015 & 0.831 & 8 \\
& $\mathrm{~N}$ & 8 & 8 & 8 & 8 & 8 & 8 & 8 \\
\hline
\end{tabular}

\section{Discussion}

\subsection{Toxic Metal Concentrations}

The levels of all but one of the toxic metals analyzed in the Lower Usuma dam water were below the WHO, NSDWQ and USEPA guidelines. The exception, $\mathrm{Pb}$ gave a reasonably higher mean concentration of $0.0993 \mathrm{mg} / \mathrm{L}$, as against the WHO limit of $0.01 \mathrm{mg} / \mathrm{L}$. In the Tilapia, Ni was slightly above limit at 0.609 $\mathrm{mg} / \mathrm{kg}$ and the level of $\mathrm{Pb}$ was significantly high at $7.109 \mathrm{mg} / \mathrm{kg}$. Ni in catfish was observed to be $0.759 \mathrm{mg} / \mathrm{kg}$ while $\mathrm{Pb}$ was $3.76 \mathrm{mg} / \mathrm{kg}$. The WHO limits for $\mathrm{Ni}$ and $\mathrm{Pb}$ in fish are $0.5 \mathrm{mg} / \mathrm{kg}$ and $1.0 \mathrm{mg} / \mathrm{kg}$, respectively (WHO, 2011). The presence of $\mathrm{Ni}$ and $\mathrm{Pb}$ in the fishes, above WHO and USEPA limits is attributed to the high level of the metals in the surrounding water and the known ability of fishes to bioaccumulate toxic metals in their tissues over time. Further investigation is needed to assess the metal speciation in the aquatic system as well as the effect of temperature and $\mathrm{pH}$ in bioaccumulation of these metals in the fish species (Ndimele et al., 2011a; Dhanakumar et al., 2015). Toxic metals of concern in the sediment were $\mathrm{Cr}, \mathrm{Pb}$ and $\mathrm{Zn}$, with the trend of toxic metal concentration in sediment being in the order: $\mathrm{Zn}>\mathrm{Pb}>\mathrm{Cr}>\mathrm{Cu}>\mathrm{Cd}>\mathrm{As}>\mathrm{Ni}>\mathrm{Hg}$.

Generally, most of the toxic metal concentrations were found to be higher in Tilapia and the sediment than water or Catfish from the dam. This highlights the benthic nature of Tilapia fish and its great capacity to bioaccumulate heavy metals (Mokhtar et al., 2009; Abdulali et al., 2012).

Sources of $\mathrm{Ni}$ and $\mathrm{Pb}$ contamination in the dam are probably due to disposal of tyres, batteries, garbage containing lead and soldered tins and fittings into the dam or its surroundings. It may also be due to contamination of any of the rivers that contribute water into the dam such as the high level of $\mathrm{Pb}$ in Guarara water transfer, as suggested by Okunlola et al. (2014).

\subsection{Ecological Risk Index}

Contamination factor $\left(C_{f}^{i}\right)$ was below one for all the toxic metals under study, in the water, Catfish, Tilapia and sediment samples from the Lower Usuma dam. This means there is generally, LOW possibility of contamination from these metals through any of the media analyzed.

Ecological risk index $\left(E_{r}^{i}\right)$ values for all the toxic metals in water and catfish were all below 30, showing slight risk of toxic metal poisoning from ingestion of water or the catfish. This indicates safety; water and catfish from the dam pose no ecological hazard to aquatic life or humans that come in contact with them. The same cannot be said of Tilapia or sediment from the dam as the ecological risk index for $\mathrm{Pb}$ in Tilapia and $\mathrm{Zn}$ in the sediment is greater than 30 but less 
than 60. This accords MEDIUM ecological risk associated with ingestion of Tilapia fish or sediment from the lower Usuma dam. The risk indices for other toxic metals in Tilapia and sediment from the dam is observed to be slight.

The overall risk index (RI) for all the toxic metals in water, Catfish, Tilapia and the sediment from the Lower Usuma dam is to be graded as A (SLIGHT), since the values are way below 40 .

\subsection{Human Health Risk Index}

The non-cancer risk or hazard quotient for $\mathrm{As}, \mathrm{Cd}, \mathrm{Cr}, \mathrm{Cu}, \mathrm{Hg}$, Ni and $\mathrm{Zn}$ were below one in water, Catfish and Tilapia thereby conferring a SAFE status for them in the media mentioned. However, the hazard quotient for $\mathrm{Pb}$ in the media was greater than one, according $\mathrm{Pb}$ an UNSAFE grade. Continued consumption of water and fish directly from the dam (without treatment) may cause health effects associated with lead poisoning (see 2.3.2) but cancer will not necessarily occur.

Total cancer risk obtained for toxic metals in water, being within the acceptable or tolerable risk for regulatory purposes of $10^{-6}-10^{-4}$ (USEPA, 2001), shows that water from the lower Usuma dam FCT poses NO cancer risk to its users, even without treatment.

Since the total cancer risk index value for toxic metals in catfish is below the acceptable or tolerable risk for regulatory purposes of $10^{-6}-10^{-4}$ (USEPA, 2001), it can also be deduced that catfish from the lower Usuma dam FCT poses NO cancer risk to its consumers.

The cancer risk for the carcinogens $\mathrm{As}, \mathrm{Cd}, \mathrm{Cr}$ and $\mathrm{Ni}$ in Tilapia and sediment samples were found to be below the tolerable limit $\left(10^{-6}-10^{-4}\right)$. Though the hazard quotient for $\mathrm{Pb}$ grades Tilapia as unsafe, since $\mathrm{Pb}$ is not classified a carcinogen, as well as $\mathrm{Cu}, \mathrm{Hg}$ and $\mathrm{Zn}$, there is NO cancer risk from their consumption in Tilapia though, $\mathrm{Pb}$ poses significant non-cancer risk.

There is no cancer or non-cancer risk posed to humans or the dam ecosystem as a whole, either by ingestion, inhalation or dermal contact with sediment from the dam. This is because the chronic hazard index for all the toxic metals, determined using the equations proposed by Wang et al. (2005), were all below one and the total cancer risk (TCR) was below the range $10^{-6}-10^{-4}$ (WHO, 2011).

\subsection{Statistical Analysis}

Table 18 presents the t-test result of the raw water and toxic metals concentration in tilapia fish from Lower Usuma Dam during the rainy season. The mean difference for raw water in rainy season stood at -0.03 with significant level of 0.65 while the mean for tilapia fish stood at 0.65 with a significant level of 0.25 which is greater than the significance level of 0.05 . Since the $t$-value for raw water stood at -0.47 with a significance level of 0.65 while the $t$ value for metal concentration of tilapia fish is 1.26 , with a significant level of 0.25 which is less than the significance level of 0.05 . It can, therefore, be concluded that toxic metal 
concentrations in the water affects the tilapia fishes in the Lower Usuma Dam during the rainy season.

Table 19 shows the summary of Analysis of Variance (ANOVA) on assessing the levels of toxic metals ( $\mathrm{As}, \mathrm{Cd}, \mathrm{Cr}, \mathrm{Cu}, \mathrm{Hg}, \mathrm{Ni}, \mathrm{Pb}$ and $\mathrm{Zn}$ ) in raw water, specifically in tilapia fish from the lower Usuma dam, Abuja, Nigeria. The calculated F-ratio is equal to 53.80 at $p$-value of 0.02 . Since $p$-value stood at 0.02 which is below 0.05 level of significance, there is significant relationship between toxic metals in Tilapia fish and raw water in Lower Usuma Dam during rainy season as such when consumed, during raining season it does not have greater effect on human.

Table 20 presents the t-test result of the raw water and toxic metals Concentration in tilapia fish from Lower Usuma Dam during the dry season. The mean difference for raw water in dry season stood at -0.11 with significant level of 0.36 while the mean for tilapia fish stood at 0.03 with a significant level of 0.60 which is greater than the significance level of 0.05 . The $t$-value for raw water stood at -0.97 with a significance level of 0.36 while the $t$ value for metal concentration of tilapia fish is 0.55 with a significant level of 0.60 which is greater than the significance level of 0.05 . Thus, toxic metal concentrations in water does affect the tilapia fishes in Lower Usuma Dam Raw Water during the dry season.

Table 21 shows the summary of Analysis of Variance (ANOVA) on assessing the levels of toxic metals (As, $\mathrm{Cd}, \mathrm{Cr}, \mathrm{Cu}, \mathrm{Hg}, \mathrm{Ni}, \mathrm{Pb}$ and $\mathrm{Zn}$ ) in water during dry season, specifically on tilapia fish from the lower Usuma dam, Abuja, Nigeria. In the analysis, the calculated F-ratio is equal to 422.31 at $p$-value of 0.02 which is below 0.05 level of significance. There is a significant relationship between toxic metals in tilapia fish and raw water in Lower Usuma Dam during dry season. The result shows that when it's consumed during dry season, it does not have greater effect on human.

Table 22 above shows the level of relationship between raw water of rainy and dry season and tilapia fish from the lower Usuma dam Abuja, Nigeria. The Pearson correlation coefficient of Tilapia fish (T F) in rainy season stood at 0.51 at a $p$-value of 0.20 which is less than the $p$-value of 0.05 . This implies that there is $51 \%$ relationship between raw water and tilapia fish during rainy season. There is an average relationship between raw water containing metal concentration and tilapia fish during rainy season from the lower Usuma dam Abuja. The table above further revealed the level of relationship between raw water and tilapia fish during dry season from the lower Usuma dam Abuja, Nigeria. The Pearson correlation coefficient stood at 0.60 at a $p$-value of 0.12 which is greater than the $p$-value of 0.05 . This implies that $60 \%$ relationship exists between raw water and tilapia fish during dry season from the lower Usuma dam Abuja. The researcher therefore conclude that there is a good relationship between raw water containing metal concentration and tilapia fish during dry season from the lower Usuma dam Abuja, Nigeria.

Table 23 presents the t-test result of the raw water and toxic metals' concen- 
tration in African Catfish from Lower Usuma Dam during rainy seasons. The mean difference for raw water in rainy season stood at -0.47 with significant level of 0.65 while the mean for catfish stood at -0.03 with a significant level of 0.81 which is greater than the significance level of 0.05 . Since the $t$-value for raw water stood at -0.47 with a significance level of 0.65 while the $t$ value for metal concentration of Catfish is -0.25 and a significant level of 0.81 which is also greater than the significance level of 0.05 . Thus, the researcher concludes that Toxic Metal Concentrations in water affect the catfish in Lower Usuma Dam Raw Water during rainy season.

Table 24 shows the summary of Analysis of variance (ANOVA) on assessing the levels of toxic metals ( $\mathrm{As}, \mathrm{Cd}, \mathrm{Cr}, \mathrm{Cu}, \mathrm{Hg}, \mathrm{Ni}, \mathrm{Pb}$ and $\mathrm{Zn}$ ) in raw water, specifically on Catfish from the lower Usuma dam, Abuja, Nigeria. In the analysis, the calculated F-ratio is equal to 0.39 at $p$-value of 0.83 . Since $p$-value stood at 0.83 which is greater than 0.05 level of significant. The researcher therefore concludes that there is no significant relationship between toxic metal in catfish and raw water in Lower Usuma Dam during rainy season. The result shows that catfish when consumed during rainy season might be harmful to humans.

Table 25 presents the t-test result of the raw water and toxic metals concentration in Catfish from Lower Usuma Dam during the dry season. The mean difference for raw water in the dry season stood at -0.97 with significant level of 0.36 while the mean for Catfish stood at -0.01 with a significant level of 0.90 which is greater than the significance level of 0.05 . The $t$-value for raw water stood at -0.97 with a significance level of 0.36 while the $t$ value for metal concentration of Catfish is -0.13 and a significant level of 0.90 which is greater than the significance level of 0.05 . It can be concluded that toxic metal concentrations in water does affect the Catfish in Lower Usuma dam raw water during dry season.

Table 26 shows the summary of Analysis of variance (ANOVA) on assessing the levels of toxic metals ( $\mathrm{As}, \mathrm{Cd}, \mathrm{Cr}, \mathrm{Cu}, \mathrm{Hg}, \mathrm{Ni}, \mathrm{Pb}$ and $\mathrm{Zn}$ ) in raw water during dry season, specifically on Catfish from the lower Usuma dam, Abuja, Nigeria. In the analysis, the calculated F-ratio is equal to 665.07 at $p$-value of 0.00 . Since the $p$-value stood at 0.00 which is below 0.05 level of significance, there is significant relationship between toxic metal in cat fish and raw water in Lower Usuma Dam during the dry season. The result shows that catfish is good for consumption and it's not harmful for human consumption.

Table 27 above reveals the level of relationship between raw water of rainy and dry season and Catfish (C F) from the lower Usuma dam Abuja, Nigeria. The correlation coefficient of Catfish (C F) in rainy season (R S) stood at 0.99 at a $p$-value of 0.00 which is less than the $p$-value of 0.05 . This implies that there is 99\% significant relationship between raw water and Catfish during Rainy season, which is an excellent relationship. The table further shows the level of relationship between raw water and Catfish during dry season from the lower Usuma dam Abuja, Nigeria. The correlation coefficient stood at 0.93 at a $p$-value of 0.00 which is less than the $p$-value of 0.05 . This implies that a $93 \%$ relationship exists between raw water and Catfish during dry season from the lower Usuma dam Abuja. 
Table 28 presents the summary of Analysis of variance (ANOVA) on assessing the levels of toxic metals ( $\mathrm{As}, \mathrm{Cd}, \mathrm{Cr}, \mathrm{Cu}, \mathrm{Hg}, \mathrm{Ni}, \mathrm{Pb}$ and $\mathrm{Zn}$ ) in sediment from the lower Usuma dam. In the analysis, the calculated F-ratio is equal to 0.00 at $p$-value of 0.00 and $p$-value stood at 0.00 , which is less than 0.05 level of significant. There is, therefore, a significant relationship between toxic metals in Catfish and raw water in Lower Usuma Dam during rainy season.

Table 29 reveals the summary of Analysis of variance (ANOVA) on assessing the levels of toxic metals ( $\mathrm{As}, \mathrm{Cd}, \mathrm{Cr}, \mathrm{Cu}, \mathrm{Hg}, \mathrm{Ni}, \mathrm{Pb}$ and $\mathrm{Zn}$ ) in sediment (Sed) in dry season (D S) from the lower Usuma dam, Abuja, Nigeria. In the analysis, the calculated F-ratio is equal to 0.00 at $p$-value of 0.00 and $p$-value stood at 0.00 which is less than 0.05 level of significance. That implies that there is a significant relationship between toxic metals and raw water in Lower Usuma Dam sediment during the dry season period.

Table 30 shows the intensity of relationship between raw water and sediment in rainy season from the lower Usuma Dam Abuja, Nigeria. The Pearson correlation coefficient of raw water and sediment during rainy season stood at 0.52 at a $p$-value of 1.9 , which is greater than the $p$-value of 0.05 . This implies that there is $52 \%$ relationship between raw water and sediment during the rainy season. Hence, there is an average relationship between raw water metal concentration and that of sediment during the rainy season from the lower Usuma dam Abuja.

The table reveals the relationship between Tilapia fish and sediment in rainy season from the lower Usuma dam Abuja, Nigeria. The Pearson correlation coefficient between Tilapia fish and sediment stood at 0.98 at a $p$-value of 0.00 which is less than the $p$-value of 0.05 . This implies that there is $98 \%$ relationship between tilapia fish and sediment during the rainy season and there is a strong relationship between tilapia fish and sediment containing metal concentration during rainy season from the lower Usuma Dam Abuja.

The Pearson correlation coefficient between catfish and sediment stood at 51 with a $p$-value of 1.99 which is greater than the $p$-value of 0.05 . This implies that there is $51 \%$ relationship between Catfish and sediment during the Rainy season which is an average relationship between catfish and sediment containing metal concentration during rainy season from the lower Usuma dam Abuja. The table revealed relationship between raw water and sediment in dry season from the lower Usuma dam Abuja, Nigeria. The Pearson correlation coefficient of raw water and sediment during the dry season stood at 0.99 at a $p$-value of 0.00 which is less than the $p$-value of 0.05 . This implies that there is $99 \%$ excellent relationship between raw water and sediment during dry season and that is a strong relationship between raw water and sediment containing metal concentration during dry season from the lower Usuma dam Abuja. The table further reveals the level of relationship between Tilapia fish and sediment in dry season from the lower Usuma Dam Abuja, Nigeria. The Pearson correlation coefficient between Tilapia fish and sediment stood at 0.69 at a $p$-value of 0.06 which is greater than the $p$-value of 0.05 . This implies that there is $69 \%$ relationship between toxic metal concentration in tilapia fish and sediment during dry season. There exists 
a slightly above average relationship between toxic metal concentration in tilapia fish and sediment during the dry season from the lower Usuma dam Abuja.

The Pearson correlation coefficient between Catfish and sediment stood at 96 with a $p$-value of 0.00 which is less than the $p$-value of 0.05 . This implies that there is $96 \%$ relationship between Catfish and sediment in the dry season. That represents an excellent relationship between toxic metal concentration in catfish and sediment during dry season from the lower Usuma dam Abuja.

\section{Conclusion}

Toxic metal concentrations of $\mathrm{As}, \mathrm{Cd}, \mathrm{Cr}, \mathrm{Cu}, \mathrm{Hg}, \mathrm{Ni}, \mathrm{Pb}$ and $\mathrm{Zn}$ in the water, African Catfish, Tilapia and sediment samples from the Lower Usuma dam Abuja were determined using Atomic Absorption Spectroscopy and Atomic Emission Spectroscopy. Ecological and human health risk indices were evaluated using the toxic metal concentrations obtained and the index values were interpreted.

As, $\mathrm{Cd}, \mathrm{Cr}, \mathrm{Cu}, \mathrm{Hg}, \mathrm{Ni}$ and $\mathrm{Zn}$ levels in the water were below WHO standard but $\mathrm{Pb}$ concentration was above limit. In the fish samples (African Catfish and Tilapia), Ni and $\mathrm{Pb}$ were present above the USEPA and WHO limits while other metals were present in low concentrations. Considering the potential toxicity of these two metals, that raises concern as the two fish species are the most consumed species by humans in the neighboring localities. $\mathrm{Pb}$ and $\mathrm{Zn}$ levels were observed to be high in the dam sediment.

Being a highly protected environment, it is unlikely that any person/organization goes to discharge metallic waste into the dam. Again, since lead pipes are not used for water transportation to/from the dam and there is no lead component in the construction of the dam, contamination must have come from one or more of the rivers whose waters are embanked in the dam. Industrial discharge into the river Usuma and the Gurara water is proposed to be chiefly responsible for toxic metal concentration in the dam.

Ecological and human health risk assessment revealed that the overall ecological risk is slight, non-cancer risk is low and cancer risk is non-existent.

It is recommended that the laboratories in the Usuma Dam Waterworks regularly check for levels of toxic metals in the treated water, to ensure that they are kept below WHO limits, before the water is distributed to the Federal Capital Territory for use.

Further research may be carried out to:

1) Find out the concentration of other toxic metals not covered in this study, and determine their levels in water, sediments and different fish species within the dam.

2) Determine variation of toxic metal concentrations in different fish organs such as the gills, liver, muscles, bones and intestines. Research can also be carried out, in addition, to find out the variations of toxic metal concentrations in sediments at different depths of the Lower Usuma dam. 
3) Assess levels of toxic metals in crops grown around the Lower Usuma dam and irrigated with water from the dam.

4) Assess the health status of humans living around the Lower Usuma dam, to find out if there are health challenges arising from toxic metal poisoning.

\section{Conflicts of Interest}

The authors declare no conflicts of interest regarding the publication of this paper.

\section{References}

Abdulali, K. A., Shuhaimi-Othman, M., \& Ahmad, A. K. (2012). Analysis of Heavy Metal Concentrations in Tilapia Fish (Oreochromis niloticus) from Four Selected Markets in Selangor, Penninsular Malaysia. Journal of Biological Sciences, 12, 138-145. https://doi.org/10.3923/jbs.2012.138.145

Amadi, A. N. (2011). Assessing the Effects of Aladimma Dumpsite on Soil and Groundwater Using Water Quality Index and Factor Analysis. Australian Journal of Basic and Applied Sciences, 5, 763-770.

Chrostowski, P. C. (1994). Exposure Assessment Principles. In D. R. Patrick (Ed.), Toxic Air Pollution Handbook (pp. 133-163). New York: Van Nostrand Reinhold.

Dhanakumar, S., Solaraj, G., \& Mohanraj, R. (2015). Heavy Metal Partitioning in Sediments and Bioaccumulation in Commercial Fish Species of Three Major Reservoirs of River Cauvery Delta Region, India. Ecotoxicology and Environmental Safety, 113, 145-151. https://doi.org/10.1016/j.ecoenv.2014.11.032

George, E., Sommer, R., \& Ryan, J. (2013). Method of Soil, Plant and Water Analysis: A Manual for West Asia and North Africa Region: 3rd Edition (pp. 133-135). Beirut: International Center for Agricultural Research in the Dry Areas.

Hakanson, L. (1980). An Ecological Risk Index for Aquatic Pollution Control: A Sedimentological Approach. Water Resources, 14, 975-1001.

https://doi.org/10.1016/0043-1354(80)90143-8

Harikumar, P. S., Nasir, U. P., \& Rahman, M. P. M. (2009). Distribution of Heavy Metals in the Core Sediments of a Tropical Wetland System. International Journal of Environmental Science and Technology, 6, 225-232. https://doi.org/10.1007/BF03327626

Ibrahim, A. Q., Onyenekwe, P. C., \& Nwaedozie, I. M. (2014). An Efficiency Assessment of Lower Usuma Water Treatment Plant in Abuja Metropolis, Nigeria. IOSR Journal of Environmental Science, Toxicology and Food Technology, 8, 46-53. https://doi.org/10.9790/2402-081224653

Izah S. C., Bassey S. E., \& Ohimain E. I. (2018). Ecological Risk Assessment of Heavy Metals in Cassava Mill Effluents Contaminated Soil in a Rural Community in the Niger Delta Region of Nigeria. Molecular Soil Biology, 9, 1-11.

Jaishankar, M., Tseten, T., Anbalagan, N., Mathew, B. B., \& Beeregowda, K. N. (2014). Toxicity, Mechanism and Health Effects of Some Heavy Metals. Interdisciplinary Toxicology, 7, 60-72. https://doi.org/10.2478/intox-2014-0009

Jonathan, B. Y., Maina, H. M., \& Maitera, O. N. (2016). Heavy Metal Pollution Assessment in the Sediments of Lake Chad, Nigerian Sector. Bayero Journal of Pure and Applied Sciences, 9, 213-216. https://doi.org/10.4314/bajopas.v9i1.33

Khan, S., Cao, Q., Zheng, Y. M., Huang, Y. Z., \& Zhu, Y. G. (2008). Health Risks of Heavy Metals in Contaminated Soils and Food Crops Irrigated with Wastewater in Beijing China. Environmental Pollution, 152, 686-692. https://doi.org/10.1016/j.envpol.2007.06.056 
Mokhtar, M. B., Aris, A. Z., \& Praveena, S. M. (2009). Assessment Level of Heavy Metals in Penaeus monodon and Oreochromis spp. in Selected Aquaculture Ponds of High Density Development Areas. European Journal of Scientific Research, 30, 348-360.

Morais, S., Costa, F. G., \& Pereira, M. L. (2012). Heavy Metals and Human Health. In J. Oosthuizen (Ed.), Environmental Health-Emerging Issues and Practice (pp. 227-246). London: IntechOpen. https://doi.org/10.5772/29869

Ndimele, P. E., \& Kumolu-Johnson, C. A. (2011a). Preliminary Study on Physico-Chemistry and Comparative Morphometric Characterization of Cynothrissa mento (Regan, 1917) from Ologe, Badagry and Epe Lagoons, Lagos, Nigeria. International Journal of Agricultural Research, 6, 736-746. https://doi.org/10.3923/ijar.2011.736.746

Ndimele, P. E., \& Kumolu-Johnson, C. A. (2012). Some Aspects of the Physicochemistry and Heavy Metal Content of Water, Sediment and Cynothrissamento (Regan, 1917) from Badagry Creek, Lagos, Nigeria. Trends in Applied Sciences Research, 7, 724-736. https://doi.org/10.3923/tasr.2012.724.736

Okunlola, I. A., Amadi, A. N., Idris-Nda, A., Agbasi, K., \& Kolawole, L. L. (2014). Assessment of Water Quality of Gurara Water Transfer from Gurara Dam to Lower Usuma Dam for Abuja Water Supply, FCT, Nigeria. American Journal of Water Resources, 2, 74-80. https://doi.org/10.12691/ajwr-2-4-1

Ong, M. C., \& Kamaruzzaman, B. Y. (2009). An Assessment of Metals (Pb and Cu) Contamination in Bottom Sediment from South China Sea Coastal Waters, Malaysia. American Journal of Applied Sciences, 6, 1418-1423. https://doi.org/10.3844/ajassp.2009.1418.1423

Osman, A. G. M., \& Kloas, W. (2010). Water Quality and Heavy Metal Monitoring in Water, Sediments and Tissues of the African Catfish Clarias gariepinus (Burchell, 1822) from the River Nile, Egypt. Journal of Environmental Protection, 1, 389-400. https://doi.org/10.4236/jep.2010.14045

Turekian, K. K., \& Wedepohl, K. H. (1961). Distribution of the Elements in Some Major Units of the Earth's Crust. Geological Society of America Bulletin, 72, 175-192. https://doi.org/10.1130/0016-7606(1961)72[175:DOTEIS]2.0.CO;2

Uluturhan, E., Kontas, A., \& Can, E. (2011). Sediments Concentrations of Heavy Metals in the Homa Lagoon: Eastern Agean Sea, Assessment of Contamination and Ecological Risks. Marine Pollution Bulletin, 62, 1989-1997. https://doi.org/10.1016/j.marpolbul.2011.06.019

Umaru, A. B., Sangodoyin, A. Y., \& Oke, I. A. (2014). The Causes and Effect of Earth Dams Failures in North Eastern Nigeria. International Journal of Engineering and Technology, 3, 2978-2985.

United States Department of Energy (USDOE) (2011). The Risk Assessment Information System (RAIS). U.S. Department of Energy's Oak Ridge Operations Office (ORO).

United States Environmental Protection Agency (USEPA) (2001). Risk Assessment Guidance for Superfund, Volume III: Part A, Process for Conducting Probabilistic Risk Assessment (EPA/540-R-02-002). Washington DC: Office of Emergency and Remedial Response, U.S. Environmental Protection Agency.

United States Environmental Protection Agency (USEPA) (2002). A Review of the Reference Dose and Reference Concentration Process (EPA/630/P-02/002F). Washington DC: Risk Assessment Forum.

United States Environmental Protection Agency (USEPA) (2009a). Guidelines for Carcinogen Risk Assessment (EPA/630/P-03/001F). Washington DC: Risk Assessment Forum.

United States Environmental Protection Agency (USEPA) (2009b). Risk Assessment Guidance for Superfund (RAGS), Volume 1: Human Health Evaluation Manual: Part F, Supplemental Guidance for Inhalation Risk Assessment, Final (EPA/540/-R-070/002). Washington DC.

United States Environmental Protection Agency (USEPA) (2011). Exposure Factors Handbook, Final ed., (EPA/600/R-09/052F). Washington DC: U.S. Environmental Protection Agency. 
USEPA (1992). Guidelines for Exposure Assessment, EPA/600/Z-92/001. Washington DC: Risk Assessment Forum.

Wang, X. L., Sato, T., Xing, B. S., \& Tao, S. (2005). Health Risks of Heavy Metals to the General Public in Tianjin, China via Consumption of Vegetables and Fish. Science of the Total Environment, 350, 28-37. https://doi.org/10.1016/j.scitotenv.2004.09.044

World Health Organization, WHO (2011). Guidelines for Drinking-Water Quality(4th ed.).

Xu, Z. Q., Ni, S. J., \& Tuo, X. G. (2008). Calculation of Heavy Metals Toxicity Coefficient in the Evaluation of Potential Ecological Risk Index. Environmental Science and Technology, 31, 112-115.

Zhang, C. (2007). Fundamentals of Environmental Sampling and Analysis (p. 109). Hoboken, NJ: John Wiley and Sons. https://doi.org/10.1002/0470120681 